\title{
Jeutsche MedizinischeWochenschrift
}

nschrift für Schriftleitung und Georg Thieme Verlag: 7000 Stuttgart. I, Postfach 732, Herdweg 63

W. Bargmann, Kicl

H. E. Bock, Tübingen

A. Butenandt, München

E. Derra, Diisseldorf

G. R. Graham, London
N. Henning, Erlangen

F. Hoff, Frankfurt

K. Kolle $†$

H. Leicher, Mainz

E. I.etterer, Tübingen
H. Mecsen, 1) iisscldorf

R. Nissen, Basel

K. H. Schäter, Hamburg

W. Scheid, Köln

G. Schettler, Heidelherg
M. Schneider, Köln

R. Schoen, Göttingen

W. Schönfeid, Heidelberg

K. Spang, Stuttgart

A. Windorfer, Erlangen

Schriftleitung: Prof. Dr. F. Grosse-Brockhoff Disscldorf

Prof. Dr. F. Kümmerle Mainz

Dr. R. H. Rosie stutgart

Inhaltsverzeichnis für den I00. Jahrgang

2. Halbjahr 1975 Hefte $27-52$

582 Abbildungen in 62.3 Einzeldarstellungen und 42.5 Tabellen

Georg Thieme Verlag · Stuttgart 


\section{Sachverzeichnis'}

A

Abrasionszytologie des Magens 1493

Absencen, atypische, SuccinimidDipropylacetat-Therapie $\mathbf{1 5 6 4}$

Acrodermatitis enteropathica, Therapie mit Zink 1549

ACTH-Produktion, ektopische, Lungenkrebs 1755

-, selektiver Mangel bei Geschwistern 2646

Adenom, autonomes, Radiojodtherapie $\mathbf{2 2 0 3}$

,,-- s. a. Schilddruise

Adenoma scbaceum 1649

Adolf-von-Baeyer-Gedenkmünzc 2369

Ajmalin-Test, WPW-Syndrom 2553

Akinese, M. Parkinson, Atemverhalten unter L-Dopa, DC-geblockt 1461

Akromegalie, BromocriptinTherapie $\mathbf{1 5 4 0}$

Aktinomykose 2131

Aldosteron im Plasma, Reninaktivität beim BartterSyndrom 2443

Alfons-Fischer-Medaille 1584

Alkohol, Gicht 1752

Alkoholabhängigkeit, chronische, neurologische Befunde $\mathbf{1 4 7 8}$

-, organisch begrüindete psychiatrische Befunde 2105

Alkylanticn, Plasmozytom, myclogene Leukämien 1961

Allergen-Aerosole, bronchialer Provokationstest $\mathbf{2 4 5 4}$

Allgemeinnarkose, Uberwachung nach 20.58

Alopecia arcata 2625

Amaurotische Idiotie 2126

Aminophyllin, Retard-Präparat, orales bei obstruktiven Atemwegserkrankungen $\mathbf{1 7 8 1}$

AMP, cyclisches, quergestreifter Muskel bei chronischer Niereninsuffizienz. 2397

Amylasämic, Makro- 1599

Amylase im Serum bei Niereninsuffizienz 2658

Androgene im Plasma, nach oralem Testosteron 1773

Androgenstoffwechsel, akute und chronische Lebererkrankungen 1881

Andrologie, Deutsche Gesellschaft für 2130

Anetodermie 1717

Aneurysma dissecans aortae, Röntgendiagnostik 1549

Angina pectoris, "PrinzmetalAngina " oder "Variant-Form " 1977

_ - Einteilung 2587
Angina pectoris, Revaskularisation, direkte chirurgische 2399

- -, Therapie der 1978

Angioneurotisches Odem 1814

Anstrengungsasthma 2367

Anthropologie, Konzept einer neuen - 1991

Antiarrhythmische Therapie, tachykarde Herzrhythmusstörung $\mathbf{1 8 1 0}$

Anti-D)-Prophylaxe, RhesusInkompatibilität 2010

Antidiabetika, orale, Entdeckung 2653

Anticpileptika, Absetzen der Therapie 1871

-, Nehenwirkungen 1904, 2365 , 2552

Antihypertensiva, Phäochromozytom, Katecholaminausschcidung 2349

Antihypertensive Therapie, Beta-Rezcptorenblocker $\mathbf{2 1 6 1}$

Antiinfektiöse Therapie, Wechsclwirkungen bei $\mathbf{2 4 9 6}$

Antikoagulanticn nach Herzinfarkt 1753

Antikoagulantientherapie, Dauer bei Gefäßßkrankheiten 2058

-, Pockenschutzimpfung 1615

Antinukleäre Faktoren, D-Penicillamin-Therapic $\mathbf{2 1 9 8}$

Antirheumatika 1550

Aortenisthmusstenose 1650

Aortenruptur 1718

Aprindin, Langzcit-Trendanalysen 1764

Approbationsordnung, neue, Erfahrungen mit 2579

Arcus lipoides 2586

Arteria lusoria (A. subclavia aberrans dextra) 1822

Artericlle Verschlulikrankheit 1687

- -, bei Bcinamputicrten 2275

- -, Diabetes mellitus 1827

Arzneimittelsicherhcit 2285

Arztberuf, Auswahl zum 1678 .

1813,2179

Arztdichte 2186

Arztrecht, Aufklïrungspflicht, Unfang der ärztlichen - 1914

-, Behandlung von Suizidpatienten gegen Willen der

Angchörigen 1812

-, Blutgruppenbestimmung bei Bluttransfusion, Sorgfaltspflicht 2409

-, Gelegenheitskonsultationen, ärttliche Schweigepflicht 1994

-, Gutachten, gerichtliche, Entschädigung bei stationärer Aufnahme nach BPflV() 2360
Arztrecht, Gutachtertätigkeit der ärztlichen Hochschullehrer 2123

-, Krankenunterlagen, Herausgabe an nachbehandelnde Arzte 2295

-, Laboratoriumswerbung, ärztliches Berufsrecht 2233

-, Numerus clausus, Entscheidung des Bundesverfassungsgerichts $\mathbf{1 7 5 0}$

-, Nutzungsentgelt, Abfuihrung von, durch ärztliche Hochschullehrer 2363

-, Rauchverbot bei Fortbildungsveranstaltungen $\mathbf{1 4 8 1}$

-, Schadenersatz wegen mißlungener Sterilisation 2657

-, Schweigepflicht, ärztliche, und Gelegenheitskonsultationen 1994

,,--- , geschichtliche Entwicklung 1867

,,,--- Neuregelung durch Strafrechtsreform $\mathbf{1 8 6 5}$

-, Zulassung zur Kassenpraxis von bcamteten und angestellten Ärzten 1613

Aspergillom 1586

-, Differentialdiagnose $\mathbf{1 8 7 0}$

Asthma, Anstrengungs - 2367

Ataxic, zcrebellare, bei Hypothyreose 1504

Atcbrin, Einführung in dic Therapic 2583

Atemwegserkrankungen, obstruktive, Pathophysiologie 1575

-, -, Retard-AminophyllinPräparat, orales $\mathbf{1 7 8 1}$

Aufklärungspllicht des Arztes, Umfrage bei Patienten 1587

Autoimmun-Thrombozytopenien, Plättchenproduktion und lienale Plättchenspcicherung 2641

AV-Block, chirurgisch induzicrt 2389

B

Bakteriurie, Nachweis antikörperbesetzter Bakterien zur Lokalisation von Harnwegsinfektionen 2598

-, Nachweis durch Blasenpunktion 2252

Bartter-Syndrom, Plasmaaldosteron und Reninaktivität 2443

BCG-Histiozytose, generalisierte, bei angeborenem Immundefekt 2604

Beckenvenenthrombose 1550

Behinderte Kinder, Statistik nach Umfrage 19742624
Belastungs-EKG und Koronarangiogramm 2538

Bernhard-Nocht-Institut 1874

Beta-Rezeptorenblocker, antihypertensive Therapie mit 2161

Bicepssehnenruptur 1615

Billroth-I-Magen, duodenaler Reflux, Gastritis 2385

Bing-Horton-Syndrom 1647

Blasenkarzinom, Urinzytologie 2340

Blasenpunktion, Nachweis einer Bakteriurie 2252

-, suprapubische 2565

Bleichmittel, kosmetische, Quecksilbervergiftung $\mathbf{1 6 9 4}$

Blcivergiftung 2303

Blindheit, kortikale, nach Vertebralisangiographie $\mathbf{2 6 2 1}$

Blutalkohol und Essig 1647

Blutspendebereitschaft 1583

Blutzucker, Anstieg nach Glucosebelastung 2587

Blutzuckerbestimmung, Reflomat-Methode $\mathbf{1 6 9 7}$

Blutzuckertagesprofil, kontinuierliches, Seruminsulin, Körpergewicht $\mathbf{1 5 9 5}$

Blutzuckerwert, postprandialer 2299

Botulinusintoxikation 2394

Brom-Verbindungen, frei käufliche, Gefährdung durch 1580, 2180

Bromocriptin, Therapic der Akromegalie 1540

Broncholytische Langzeittherapie, orale, Theophyllin-Äthylendiamin, Lungenfunktion 2482

Butylphenol, paratertiäres, Vitiligo, Hepatosplenopathie und Struma nach Arbeit mit 2216

B-Vitamine, therapeutische Wirkung bei chronischer Hepatitis 2074

C

Cabot-Ring 1757

Cacrulein, Dünndarmpassage 2488

Calciumheparinat, Thromboseprophylaxe bei Hüftgelenksoperationen 2014

Calciumtransport, intrazellulärer, 1854

Carus-Medaille 2369

Chemotherapie, adjuvante, bei soliden Tumoren 2342

-, kombinierte, therapieresistente Leukämien des Erwachsenen 2478

"China-Restaurant-Syndrom 2126 
Chloracetat-Esterase-Rcaktion, Eosinophilenleukämic 2092

Chloramphenicol, Indikationen für 1482,1679

Cholangiographic, cndoskopischradiologische, Cholestase, PostcholezystektomicSyndrom 1877

Cholelithiasis, Tiphusdauerausscheider 1714

Cholera, Fntdeckung des Frregers 2119

Cholestase, endostopischradiologische (holangiographic 1877

-, Nachweis von Lipoprotein X 2193

Cholestcrin-Smintigraphie, ${ }^{1: 31} \mathrm{~J}-$, Nebennieren 2524

Chondromatosis synovialis 2183

Chromatinfibrillen, Aufbau der 1818

Chromosomen, Untersuchungen an Lymphozyten bei multiple Sklerose 2028

Clofibrat, Serumlipide 2486, 2624

Clostebol bei chronischer Hepatitis 2074

CO-Vergiftung 2240

Coma diabeticum, niedrig dosierte Insulintherapie $\mathbf{1 9 9 5}$

Cor pulmonale und Digitalisintoxikation 2002

Corticosteroide, Wirkung bei Lymphogranulomatose 2183 Cothenius-Medaille 2369

Cyclophosphamid, Synchronisationstherapie hei malignen l.ymphomen 1719

\section{D}

Dehydratation, hyperosmolare, im Kindesalter 1479

Diabetes, juveniler 2000

Diabetes mellitus, arterielle Verschlulikrankheit $\mathbf{1 8 2 7}$

- -, Mammakarynom, metastasierendes 2422

- -, Ovulationshemmer 2411

- -, Sympathektomic 2058

- -, Virusätiologic 1997

Diarrhoe, chologene, Ursachen der $\mathbf{1 6 3 9}$

Digitalis, Alter 2510

Digitalis, Herzinfarkt, akuter 2417

-, Herztherapic mit, seit 200 Jahren 1980

-, Kontraktilität der insuffizienten Ventrikel unter Belastung 2265

Digitalisintoxikation, Serumglykosidkonzentration $\mathbf{1 7 6 8}$

-, und Cor pulmonale 2002

Digoxin, biologische.Verfügbarkeit bei Billroth II-Magen 2430

Dihydroergotamin, postoperative Thromboembolieprophylaxe 2065
Diphenylhydantoin-Intoxikation, Fosinophilenleukämic, akute Polyneuropathic 1532

Diphtheric-Schutzimpfung, Entwicklung durch Emil von Behring 2172

Diuretika-Therapie, Na: K()uoticnt im Urin 1918

DMW - 100 Jahre

-, Antidiabetiki, orale, Entdeckung 2653

-, Arebrin, Einfuihrung des 2583

-, Cholera-Erreger, Entdeckung des 2119

-, Fmil von Bchring, aktive und passive Schutrimpfung 2172

-, Haemophilus influenzac, Frstbeschreibung durch Richard Pfeiffer 2405

-, Maul- und Klauenseuche, Entdeckung des Virus der 2355

-, Robert Koch, seine Bedeutung für dic Tropenmedizin 1933

-, Röntgenstrahlen, Einführung in dic Medizin 2457

-, Rotz-Bazillus, Entdeckung des 2292

-, Schutzimpfung, aktive und passive, Fmil von Behring 2172

-, Sulfonamide, Entdeckung de 2617

-, Tetanusbarillen, Entdeckung der 2230

-, Tuberkelbazillen-Färbung, Entdeckung der 2055

-, Tuberkulin, Entdeckung des 1925

-, Ultraviolettlicht, Ultraschall, Einführung in die Medizin 2549

-, Wassermannsche Reaktion, Fntwicklung der 2506

L-Dopa, deciarboxylasegeblockt, Atemverhalten von Parkinson-Kranken $\mathbf{1 4 6 1}$

-, -, - Therapie, Nebenwirkungen 2165

Dünndarmpassage, Cacrulein 2488

Dünndarmschleimhaut, digestiv-resorptive Funktion 1603

\section{E}

Fchokardiographie, asymmetrische Septumhypertrophie $\mathbf{2 1 8 9}$

Econazol, poliklinische Priifung 1497

-, vulvo-vaginale Mykose $\mathbf{1 7 3 0}$

Ecthyma contagiosum 2515

Einwanderungsbeschränkung für ausländische Ärzte in USA 1756

Emphysem, retrobulbäres, und frontale Schädelfraktur 2304

Endokarditis, akute bakterielle 2589

Enteroviren, Persistenz von Antikörpern in der gesunden Bevölkcrung $\mathbf{1 6 6 3}$ Naphthol-AS-D-chloracetatFsterase-positive Granula, beim Kind 2092

Ernst-von-Bergmann-Plakette $1680,2414,2624$

Ernst-Klenk-Stiftung 2062

Frregungsrückbildungsstörungen, unspezitische 1646

Frythemal e calore 2415

Frythematodes, systemischer 2555

Erythrozyten-Antikörper. irreguläre, Häutigkeit 1567. 1712

Fssig und Blutalkohol 1647

Ftotibrat, Serumlipide 2486 , 2624

Exfoliativ-Zytologie, Tuhenkarzinom-Frïherkennung 1476

Fxostosen, multiple kartilaginärc, Kolon- und MagenPolypose 2316

F

Faserembolien, Komplikationen bei Eingriffen am Herzen 1551

Feinnadelbiopsie, Metastasenleber $\mathbf{2 6 0 2}$

Fettleber, alkoholische bei Primaten 1616

Fibrinolyse, arterielle Verschlußkrankheit, Phlebothrombose 1687

Ficher, Abwehrmechanismus 1805

Fludrocortison, orthostatische Kreislaufregulationsstörung Ophthalmodynamographie 1790

Fornixdivertikel 2516

\section{G}

Gastritis, Billroth-1-Magen, Reflux 2385

Gastrostomic-Ernährung Osophagusersat\%plastik nach 2462

Galaktographic, Mammakarzinom-Frühdiagnostik 1916

Gallensteinauflësung in vivo 1619

Gangrän, Wirkung von Vasodilatatoren 2658

Gegenpulsation, intra-aortale 1968

Gentamicin, Vergleich mit Tobramycin, Sisomicin $\mathbf{2 1 4 4}$

Gerinnungsstörungen beim Lyell-Syndrom 2337

Gieschlechtskrankheiten, Erkrankungsziffer 19741756

Gicht und Alkohol 1752

Gichttophi, Rückbildung von 2183

Giftgas, Lungenkrebs 1483

Glaukombehandlung, konservative 1919

Glibenclamid, Vergleich mit Gliquidon 1733
Gliom bei tuberöser Hirnsklerose 1553

Gliquidon, Wirkungsmechanismus 1733

Glomerulonephritis 1551

-, Formen der 1800

-, primäre, spezielle Pharmakotherapie 1803

Glucosebelastung, Blutzuckeranstieg 2587

Gonarthrose, Gelenkersatz bei 2411

Gruppenunterricht, klinischer 2545

Ginäkomastic 2612

-, Therapie der 1741

$\mathrm{H}$

Haarausfall, Quecksilbervergiftung 1694

Hämagglutination, indirekte, Vergleich mit indirekter Immunfluoreszenz, chronische Pyelonephritis $\mathbf{2 0 8 6}$

Hämoperfusion, Kohle-, bei Knollenblätterpilzvergiftung 2509

Hämophilic A, Gelenkblutungen, ambulante Substitutionstherapie 1523

Hacmophilus influenzae, Erstbeschreibung 2405

Hämorrhagische Diathesen, clektrische Impedanz-Messung, Suchmethode 1657, 1916

Haferflocken, Reduktionsdiät 2658

Haftpflicht für Ärzte, USA 1648

Harnwegsinfektion, Lokalisation, Nachweis antikörperbesetzter Bakterien im Urin 2598

- bei Kindern 2636

Heparin, subkutan, zur Thromboseprophylaxe 2014

Hepatitis A, anikterische 2553

- -B-Antigen, Nachweis von 2464

-, chronische, B-Vitamine und Clostebol 2074

- bei Primaten 1616

-, Übertragung 1515

,-- , durch Hochdruckinjektion 2465

Hepatosplenopathie nach paratertiärem Butylpheno 2216

Herpes simplex, rezidivierender, Levamisol 2510

Herzinfarkt, Anteroseptalinfarkt mit Rechtsschenkelblock 1554

-, Antikoagulantien nach 1753

-, Intensivpflege 2588

-, junger Männer 2129

-, serumlipidsenkende Pharmaka 1611

-, akuter, Digitalis bei $\mathbf{2 4 1 7}$

-, -, Nitroglycerinwirkung 2003

-, -, Verlauf und Prognose bei alten Menschen $\mathbf{2 1 3 3}$

Herzinsuffizienz, bradykarde, Schrittmacherimplantation 2070

Herzmyxome 2270 
Herzhythmusstörung, durch Heparin 2182

Herzrhythmusstörungen nach Schleudertraumen 2623

-, tachykarde, antiarrhythmische Therapic $\mathbf{1 8 1 0}$

Herzschrittmacher, Verlängerung der Funktions\%cit 1683

Herzschrittmacherimplantation, Reizschwellenverhalten $\mathbf{2 1 5 0}$

Herztöne, kindliche, Kontrolle 1482

Hidradenoma papilliferum 2187

Hippokrates-Medaille 1484

Hirnabseds, bakterielle Meningitis 2234

Histotechnologic, USA 2468

Hodenatrophic bei chronischen Leberkrankheiren 2465

Hiiftgelenksdysplasic 1997

Hüftgelenksoperationen, Thromboseprophylaxe mit Calciumheparinat 2014

Hydroxyharnstoff, Chemotherapic beim soliden Neurosirkom 2102

Hypernephrom, inoperables, Embolisation durch intraartericlle Thrombinapplikation 2428

Hyperosmolare Dehydration bei Kindern 1479

Hyperostose, sterno-kostoklavikuläre 1519

Hyperoxilurie, erworbene, Nephrolithiasis 1509

Hyperthermic, maligne 1974

Hyperthyreose, $T_{3^{-}}$, exzessive Thyroxin $\left(\mathrm{T}_{4}\right)$-Konversion 2319

Hypertonic, Blutdrucksenkung, therapeutische 1874

-, essenticlle, Prindolol 1726

Hypoglykämicsyndrom, kindliches, diffuse nesidioblastische Inselhyperplasic $\mathbf{1 9 0 6}$

Hypokaliämic 1614

Hypoparathyreoidismus, chronischer sekundärer, und Pscudotumor cerebri 2213

Hypothyreose, zerebellare Ataxic $\mathbf{1 5 0 4}$

I

IHSS, echokardiographischdiagnostische Kriterien 1759 Immundefekt, angeborener, generalisierte BCGHistiozytose 2604

Immundefektc bei Kindern, Therapie $\mathbf{1 7 1 1}$

Immunfluoreszenz, indirektc, Serodiagnostik der chronischen Pyelonephritis 2086

Immunsuppressiva, Lupus erythematodes visceralis $\mathbf{2 5 9 1}$

Impedanz-Mcssung, elektrische, bei Blutgerinnungsstörungen 1657, 1916

Infektionskrankheiten, Epidemiologie 2401
Influenza-Lebendimpfstoff, attenuierter 2629

Insclhyperplasie, diffuse nesidioblastische, kindliches Hypoglykämicsyndrom 1906

Insulin, Dosierung bei Diabetes 2623

-, - im Coma diabeticum 1995

- Körpergewicht, kontinuierliches Blutzuckertagesprofil 1595

-, nicdere Dosicrung 2623

Intensivpflege, Herzinfarkt 2588

Intrauterimpessare, Kompli-

karionen durch 2513

Intubationsnarkose, Uber-

wachungsdauer nach 2058

Isosorbiddinitrat, akuter

Mrokardinfarkt 2003

\section{J}

Jodmangel, alimentärer, in der BRD 1937

${ }^{131} \mathrm{~J}$-Cholesterin-Szintigraphic, Nebennicren 2524

\section{K}

Kalia-Azar (viszcrale l.cishmaniose) 2022

Kammerflimmern, DemandSchrittmacher 2439

Kardiomyopathic, Lupus-, bei unkompliziertem LE 2138

Karrinoid 2163

Katccholamine, Ausscheidung unter Antihypertensiva, Phäochromozytom 2349

Kaverne, Differentialdiagnose der - im Thoraxbild $\mathbf{1 7 4 2}$

Kavität, Thoraxbild $\mathbf{1 7 4 2}$

Keimbesiedlung bei Neugeborenen 1918

Kcimzahlbestimmung im Mittelstrahlurin 1614

Kinder, behinderte 2624

Knochenmarksuntersuchung, Stermalpunktion oder Myelotomic 1872

Knollenblätterpilzvergiftung 1714

-, Kohle-Hümoperfusion 2509

Kolon, Syndrom des angeborenen kleinen linken - 2554

-, irritables 2610

Kolonpolypen 1917

-, chirurgische Therapie 1672

Kolon-Polypose, multip!e kartilagin.̈̈re Exostosen bei 2316

Kontaktlinse als Therapeutikum 2572

Kontrazeptiva s. a. Ovulationshemmer

-, Gefäßkrankheiten 2058

Koronarangiogramm, BelastungsEKG, Beziehungen zwischen und 2538

Koronarangiographic, Komplikationsraten $\mathbf{1 8 6 3}$
Koronararterien, Verteilungsmuster, Einbau in das Myokard 2448

Krankenhaushetten, Überschuß in USA 2413

Krankheitsstatistik 1921

Kreatinin, Erhöhung im Serum 1583

Krebshäufigkeit bei Mormonen 2624

L

L.angerhanssche Inseln, Implantation 2238

Laparotomic, explorative, bei Lymphogranulomatose $\mathbf{1 4 8 7}$

Laser-Koagulation, endoskopische, $\mathbf{1 6 7 8}$

L.ebensmittel, Tiefgefrieren 2511

L.eber, Metastasen-, zytologische Untersuchungen 2602

Lebererkrankingen, akute, chronische, Androgenstoffwechsel und Leydig-Zellfunktion 1881

-, Phenolisatin-induzierte 2530

Leberkarzinom, Klinik des $\mathbf{1 6 2 5}$

-, nach Remission ciner akuten Lymphoblastenleukämie $\mathbf{1 7 9 2}$

Leberkrankheiten, chronische, Hodenatrophie boi 2465

-, -, Lipoproteinmuster $\mathbf{2 1 5 6}$

Leberschäden, arıncimittelbedingte, Richtlinien zur histologischen Beurteilung 1746

Lebertransplantation 2047

Leberzirrhose bei Primaten 1616

Leukämic, akute lymphatische, im Kindesalter, Erhaltungstherapie $\mathbf{2 1 6 9}$

-, chronisch-myeloische, Mamma karzinom, gleich\%citiges Vorkommen 2568

-, Eosinophilen- 2092

-, myelogene, nach Alkylanticntherapie eines Plasmozytoms 1961

-, therapieresistente, des Erwachsenen kombivierte Chemotherapie 2478

Levamisol, entzündlichrheumatische Erkrankungen, Sarkoidose 2297

-, Hautkrankheiten 1710

-, Herpes simplex 2510

-, Melanom, malignes 2510

Leydig-Zellfunktion, akute und chronische Lebererkrankungen $\mathbf{1 8 8 1}$

Linkshändigkeit 2511

Lipide im Serum 2624

Lipoproteine, bei chronischen Leberkrankheiten $\mathbf{2 1 5 6}$

- X, Nachweis bei Cholestase 2193

Liquorproteine, Pathophysiologie $\mathbf{1 6 3 0}$

Lown-Ganong-Levine-Syndrom 2556

-, Diagnostik 2536

Lues II 2371
Lues III (Gummata) 2001

Lungenatelektase, totale 1518

Lungenembolic,

Perfusionsszintigramm 1715

Lungenerkrankungen, obstruktive $\mathbf{2 2 9 8}$

Lungenfunktion, TheophyllinAthylendiamin, orale LangzeitBroncholyse $\mathbf{2 4 8 2}$

Lungeninfiltration, akute heidseitige, Komplikation der zytestatischen Therapie 2098

Lungenkrebs, cktopische AC.TH-Produktion 1755

-, Giftgas 1483

Lupus ery thematodes discoides 1875

- - disseminatus, Lupuskardiomyopathie 2138

- -, Nervensystem-Frihmanifestation 2328

- -, viszcralis, Klassifikation, immunsuppressive Therapie 2591

Luxation, perilunäre 2064

Lyell-Syndrom, Gerinnungsstörungen 2337

Lymphangiomyomatose, generalisierte 2277

Lymphknotenentfernung, prophylaktische, bei Melanom 2659

Lymphknoteninfarkt mit fieberhafter Erkrankung 1680

Lymphogranulomatose s. a. Morbus Hodgkin

-, Corticosteroidwirkung 2183

-, explorative Laparotomic, Splencktomic 1487

-, Synchronisationstherapic mit Vincristin, Cyclophosphamid, Phase-II-Studie $\mathbf{1 7 1 9}$

Lymphome, kutane, T- und B-L.ymphozyten 2562

Lymphosarkom, VincristinCyclophosphamid-

Synchronisationstherapie $\mathbf{1 7 1 9}$

Lymphosarkome, kindliche, Therapie 1548

Lymphozyten, T- und B-, kutane Lymphome 2562

M

Magenbezoare, Therapie $\mathbf{1 6 0 2}$

Magenfrühkarzinom, in Europa 1946

Magenkarzinom, Ernährung 1515

Magen-Polypose, multiple kartilaginäre Exostosen bei 2316

Magenresektion nach Billroth II, biologische Verfügbarkeit von Digoxin bei 2430

Magenschleimhaut, Dyspl.ısien 1950

Magenuntersuchung, röntgenologisch, Doppelkontrastmethode 2226

Makroamylasämie 1599 
Mammakarzinom, Galaktographic 1916

-, Leukämie, chronisch-myeloische, gleichzeitiges Vorkommen 2568

-, mctastasierendes, und

Diabetes mellitus 2422

-, Sensibilitätstestung gegen

Zytostatika in vitro 2035

-, Stadium I 1714

Masernschutzimpfung nach

Virusmeningitis 2059

Mastozytose 1617

Maul- und Klauenseuche, Entdeckung des Virus 2355

Medizinstudenten, amerikanische, Studium im Ausland 2186

Melanom, malignes, Levamisol 2510

-, - Lymphknotenentfernung, prophylaktische 2659

Mendel-Medaille 2369

Meningitis, baktericlle, und Hirnabszeł 2234

Mesaortitis luica, Corticoide 1582

Migräne, Intervallbchandlung 2464

Mikrionssynkope 2234

Mittelstrahlurin, Keimzahlbestimmung 1614

Morbus Crohn, Häufigkeit, Pathogenese 1679

-, haemolyticus neonatorum 1918

- Hodgkin, Laparoskopic 1711

- -, Prognose und Therapic im Kindesalter 2246

- -, Therapic 2411

- Köhler II 1924

- Parkinson, Atemverhalten unter L-Dopa, DC-geblockt 1461

- Pringle (Adenoma scbaceum) 1649

- Werlhof, akuter 2510

Mormonen, Krebs bei 2624

Multiple Sklerose, Chromosomenuntersuchungen an Lymphozyten $\mathbf{2 0 2 8}$

Myasthenia gravis pseudoparalytica, Therapie der $\mathbf{2 2 8 3}$

Myelom, multiples 1485

Mykosen, vulvo-vaginal, Econazol 1730

Myokardinfarkt, akuter, prognostischer Index 2517

- s. Herzinfarkt

Myokard-Szintigraphie mit Thallium-201 2373

$\mathrm{N}$

Nagelverfärbung, Quecksilbervergiftung 1694

Naphthol-AS-D-chloracetatEsterase-positive Granula, Eosinophilenleukämie 2092

Narkose, Allgemein-, Uberwachung nach 2058

Nasopharynxkarzinom, okkultes 2527
Natrium-Kalium-Quotient im Urin 1918

Natrium-Kreatinin-Quotient in Harn, Plasmareninaktivität, Nomogramm 2649

Nebennieren-Szintigraphie mit 1:31 J-Cholesterin 2524

Necrobiosis lipoidica diabeticorum 1517

Neoplasien im Kindesalter, maligne, Radio-Chemotherapie des Zentralnervensystems 1651

Nephrolithiasis und Hyperoxalurie, erworbene 1509

Nephropexic bei Senknicre 1501

Neuroleptika, trizyklische,

Patienteninformation, Blutbildkontrollen 1715

Neuro-Phospholipidose, kutane, als Arzneimittelnebenwirkung und bei malignen metastasierenden Tumoren 1809

Neurosirkom, solides, Chemotherapic mit Hydroxyharnstoff 2102

Nicreninsuffizienz, Hämostase bei 2299

-, chronische, CAMP-Gehalt des quergestreiften Muskels 2397

-, Scrumamylase bei 2658

Nierentransplantation bei Kindern 1641

-, Pilzinfektion nach 2082

Nitroglycerin, akuter Myokardinfarkt 2003

Nobelpreis für Medizin 1975 2302

Normann-Medaille 2370

\section{$\mathrm{O}$}

Obduktion, 1600 Jahre nach dem Tod 2000

Osophagusersat\%plastik, Gastrostomic-Ernährung 2462

Osophagusvarizenblutung, Pneumothorax als Therapicversuch 2125

Ohrgeräusche 1753

Onkozytom 2063

Ophthalmodynamographie, Fludrocortison bei orthostatischen Beschwerden 1790

Orthostatische Kreislaufregulationsstörung, Fludrocortison bei $\mathbf{1 7 9 0}$

Osteopoikilie 2416

Ovulationshemmer, Diabetes mellitus 2411

-, Glucosestoffwechsel 1703

\section{P}

Panarteriitis nodosa 2018

Pankreas, künstliches 1482

Pankreasszintigraphie 1465

Pankreatitis, akute hämorrhagisch nekrotisierende, vorzeitige Operation bei 2241

Parasitenübertragung durch Endoskope 2366

Parotis, Sialadenose $\mathbf{1 5 4 5}$
Paul-Langerhans-Plakette 1616

Pemphigus chronicus benignus familiaris 1585

D-Penicillamin, Nebenwirkungen 1634

-, -, -Präparate, Verträglichkeit 1646

-, -, Therapie, antinukleäre

Faktoren 2198

Perfusionsszintigramm, Lungenembolie 1715

Perikarditis, akute 1618

Peronacusparese, periphere, Therapie bei 1483

Petit mal, posttraumatisch 2366

Pflegehilfe 2414

Phäochromozytom 2469

-, Antihypertensiva, Katecholaminausscheidung 2349

-, Herzgeräusch, diastolisches 1530

Pharmaka, Metabolismus von -, Arzncimittelsicherheit $\mathbf{2 2 8 5}$

Phenacetin, Nierenschädigung 1451, 1709, 2057

Phenolisatin, Lebererkrankungen 2530

Phenprocumon (Marcumar $\left.{ }^{(}\right)$, Mordversuch mit $\mathbf{1 8 3 8}$

Phenylbutazon in der Stillzcit 2366

Philipp-Stöhr-Medaille 2370

Phlebothrombose, Fibrinolyse 1687

Phospholipidose, kutane Neuro-, als Arzneimittelnchenwirkung und bei malignen metastasicrenden Tumoren 1809

Photochemotherapic, Psoriasis 2471

Pilyinfektionen nach Nierentransplantation 2082

Plasmareninaktivität, Prindolol bei essentieller Hypertonic $\mathbf{1 7 2 6}$

Plasmozytom, Alkylantien, myelogene Lcukämien 1961

-, intravenöse Urographie 1813

Plazentalokalisation 1482

Pneumothorax $2188^{\circ}$

-, Therapieversuch bei Osophagusvarizenblutung 2125

Pockenimpfpflicht, allgemcine, Aufhebung der 2000

Pockeninfektiosität und Impfschutz 2062

Pockenschutzimpfung und Antikoagulantientherapic 1615

Poliklinik, medizinische, Universität Basel, 100jähriges Jubiläum 2238

Polyarthritis, chronische, testpsychologische Untersuchungen 1833

-, juvenile chronische 2043

Polyneuropathie, akute, durch DiphenylhydantoinIntoxikation 1532

Polypektomie, endoskopische, im oberen Verdauungstrakt 2313

Postcholezystektomie-Syndrom endoskopisch-radiologische Cholangiographie $\mathbf{1 8 7 7}$

Preisausschreibungen Alexander-Schmidt-Preis 2062 Angiologisches Stipendium 1516

Anna-Monika-Stiftung 2238

"Biochemische Analytik"Preis 1820

C. E.-Alken-Preis 1921

Curt-Adam-Preis 2414

Dr.-Martini-Stiftung 1874

Edens-Preis 2186, 2554

Erich-Krieg-Preis 1874

Erlanger Förderungspreis 2659

Ernst-Hutzenlaub-Tierschutz-

Forschungspreis 1584

Felix-Wankel-Tierschutz-

Forschungspreis 1584

Ferdinand-Bertram-Preis 1820

Förderpreis der Deutschen

Diabetes-Gesellschaft $182 \mathrm{C}$

Franz-Redeker-Preis 2660

Fricdrich-von-BodelschwinghPreis 2186

Gerhard-Domagk-Preis 1976 1516

Hans-Liniger-Preis 2660

Hermann-Simon-Preis 1584

Hufeland-Preis 19761584

Internationaler Preis für moderne Ernährung 2660

Johann-Georg-ZimmermannFörderpreis für Krebsforschung 1584

Kncipp-Preis 2660

Kneipp-Preis fuir "Gesundheitshildung " 1584

Ludolf-Krehl-Preise 2659

Max-Ratschow-Preis 2660

Paul-Martini-Preis 2660

Professor-Franz-von-BrückePreis 1516

Robert-Feulgen-Preis 1874

Stadt Bad Kissingen, Preis der 2660

Stiftung Michael 1874

Theodor-Frerichs-Preis 2062

Theodor-Naegeli-Preis 2660

Dr.-Willmar-Schwabe-Preis 2660

Preisverleihungen

Adalbert-Czerny-Preis 2369

Albrecht-von-Graefe-Preis 2468

Alexander-Schmidt-Preis 1716

Alexandre-Besredka-Preis 2554

Alfred-Vogt-Preis 19752370

Arnold-Biber-Preis 1648

Arthur-Pappenheim-Preis 2468

Avery-Lindsteiner-Preis 2369

Bertram-Preis 1484

Curt-Adam-Preis 1552

Carol-Nachman-Preis 1552

Dr.-Dora-Seif-Preis 1820

Dr.-Martini-Preis 1484, 1648

Dr.-Willmar-Schwabe-Preis 2588

E.-K.-Frey-Preis 2370

Erich-Lexer-Preis 1484

Feldberg-Preis 2370 
G.-E.-Konjetzny-Preis 1484 Georg-von-Hevesy-Preis 2514

George-E.-Shambaugh-Preis 2468

Gödecke-Forschungspreis 1874

Gustav-Riehl-Preis 1648

Henry-E.-Sigerist-Preis 2468

Homburg-Preis 1716

Homer-W.-Smith-Award in

Renal Physiology 19752370

Hugo-Spatz-Preis 2554

Jubiläumspreis der Firma

B. Braun-Melsungen AG 1484

Karl-Hanser-Gedächtnispreis 2514

Ludolf-Krchl-Preis 1648

Ludwig-Darmstädter-Preis 1921

Ludwig-Heilmeyer-Medaille in Gold 2514

Ludwig-Heilmeyer-Medaille in Silber 2514

Ludwig-Rehn-Preis 2554

Martin-Wassmund-Preis 1716

Max-Bürger-Preis 1820

Max-Cloëtta-Stiftung 2624

Michael-Preis 1552

Minkowski-Preis 1921

Nobelpreis für Medizin 1975 2302

Otto-Loos-Preis 2588

Paul-Ehrlich-Preis 1921

Paul-Martini-Preis 1648

Paul-Martini-Medaille in Gold 2370

Paul-Martini-Medaille in Silber 2624

Redel-Preis 19752370

Rober-Bing-Preis 2624

Robert-Fculgen-Preis 1874

Robert-Koch-Preis 1921, 2370

Röntgen-Preis 1648

Romano-Guardini-Preis 1975 1680

Rudolf-Schülke-Preis 1716

Schweizerische Gesellschaft für Innere Medizin, Preis der 2588

Spencer-Lashley-Preis 1975 2370

Thannhauser-Preis 2370

Thiersch-Preis 1820

Primatenforschung, USA 2130

Prindolol, Hypertonie

essentielle 1726

Prinzmetal-Angina 1977

Prostatakarzinom,

Frühdiagnose 1573

-, Metastasen 2590

Provokationstest, bronchialer, Allergen-Aerosole $\mathbf{2 4 5 4}$

Pseudo-LE-Syndrom, Arzneimittelinduzierbarkeit (Venopyronum-Dragees) 1555,1558 , 1590

Pseudomonas-Infektionen 1701

Pseudotumor cerebri, sekundärer Hypoparathyreoidismus $\mathbf{2 2 1 3}$

Psoriasis, Photochemotherapie 2471

Psychiatrische Forschung, Beitrag der Verhaltensbiologic 2108
Purpura anaphylaktoidea Schönlein-Henoch 1449

Pyelonephritis, chronische, Serodiagnostik mit indirckter Immunfluoreszenz. 2086

\section{Q}

Quecksilbervergiftung, kosmetische Bleichmittel 1694

Quinckesches Ödem 1814

\section{$\mathrm{R}$}

Radiojodtherapie, autonomes Adenom 2203

Ratschow-Gedächtnismedaille 2370

Rechtsschenkelblock mit überdrehtem Linkstyp 2125

Reduktionsdiät mit Haferflocken 2658

Reflomat, Blutzuckerbestimmung 1697

Reflux, duodenaler, Billroth-IMagen, Gastritis 2385

Rektosigmoidkarzinom, präoperative Strahlenbehandlung 2651

Rektumkarzinom, präoperative Strahlenbehandlung 2651

Renin, Plasma-Aktivität, Schätzung nach $\mathrm{Na}$-KreatininQuotient im Harn 2649

Reninaktivität, Bartter-Syndrom, und Plasmaaldosteron 2443

Renin-Bestimmung, Plasma2553

Respiratorische Insuffizienz, Sauerstofftherapie 1814

Restharnbestimmung, katheterlose 2565

Retikulumzcllsarkom, VincristinCyclophosphamid-Synchronisationstherapie $\mathbf{1 7 1 9}$

Rhesus-Inkompatibilität, Anti-D-Prophylaxe 2010

Rheumatoide Arthritis, Schilddrüsenfunktionsstörungen 2300

Rieder-Medaille 1616

Röntgenstrahlen, Einführung in die Medizin 2457

Rotz-Bazillus, Entdeckung 2292

Rubcolen-Embryopathie 2299

Ruickstandsuntersuchungen, Schlachtticre 1552

\section{S}

Sarkoidose 2574

Sauerstoffpartialdruckmessung, transkutane, pädiatrische Intensivmedizin 1892

Schädelfraktur, frontale und retrobulbärcs Emphysem 2304

Scheidungshäufigkeit 1648

Schenkelblock, bilateraler, mit AV-Block I. Grades 1486

Schilddrüse, autonomes Adenom 2223

Schimmelpilztoxine 2466

Schleiden-Medaille 2369

Schleudertraumen der Halswirbelsäule, Herzrhythmusstörungen 2623
Schock, scptisch-toxischer $\mathbf{1 6 6 7}$

Schrittmacher, Defektserie

Cordis/Stanicor 1869

-, Demand-, Kammerflimmern

bei Funktionspruifung $\mathbf{2 4 3 9}$

-, Verlängerung der Funktionszeit 1683

Schrittmacherimp!antation, bradykarde Herzinsuffizienz 2070

-, Reizschwellenverhalten $\mathbf{2 1 5 0}$

Sektion ohne Zustimmung 1998

Senkniere, NephropexieSpätergebnisse $\mathbf{1 5 0 1}$

Septumhypertrophie, asymmetrische, Echokardiographie 2189

Serratia-marcescens-Sepsis bei Kindern 2324

Serumhepatitis

s. a. Hepatitis B

Serumhepatitis-Antigen 1713

Serumlipide, Myokardinfarkt, -senkende Pharmaka 1611

Sialadenose der Parotis $\mathbf{1 5 4 5}$

Sinuatrialer Block Il. Grades 2470

Sinusknotensyndrom, sinuatrialer Block II. Grades Typ 12372

-, Sinusknotencrholungszcit 2305

Sir-Hans-Krebs-Medaille 2370

Sisomicin, vergleichende Pharmakokinetik mit Gentamicin, Tobramycin $\mathbf{2 1 4 4}$

Skorbut 1923

Somatostatin, betazol-stimulierte Magensekretion 1797

-, carbachol-stimulierte Pankreassekretion 1797

-, Gallenblasenkontraktion $\mathbf{1 7 9 7}$

Splencktomie, bei Lymphogranulomarose $\mathbf{1 4 8 7}$

Status asthmaticus, ACTH Corticoide 1753

Sterilität, Aufbewahrung sterilisierter Kanülen 1872

Steroid, anaboles (Clostebol), therapeutische Wirkung bei chronischer Hepatitis 2074

Stipendium, angiologisches 1516

Stock-Spielmeycr-Vogt-Syndrom 2126

Stoffwechselkrankheiten, hereditäre, pränatale Diagnostik 1674

Strahlentibrose, venöse Abfluß stauung 1582, 2180

Streptokinase, fibrinolytische Langzeittherapie $\mathbf{1 8 8 7}$

Struma diffusa nach paratertiärem Butylphenol 2216

Subaortale Stenose, idiopathische hypertrophe, echokardiographische Kriterien $\mathbf{1 7 5 9}$

Subarachnoidalblutung 1876

Sulfonamide, Entdeckung der 2617

Sympathektomie, Diabetes mellitus 2058

Syndrom, Bartter- 2443

-, Hypoglykämie, kindliches 1906

Syndrom, Karzinoid- 2163

-, Lown-Ganong-Levine 2536, 2556

-, Lyell-, 2337

-, Postcholezystektomie-, 1877

-, Pseudo-LE-, 1555, 1558, 1590

-, WPW-, 2536, 2553, 2626

Szintigraphie, Myokard-, mit Thallium-201 2373

-, Nebennieren-, mit ${ }^{1: 11} \mathrm{~J}$-Cholesterin 2524

-, Pankreas- 1465

$\mathrm{T}$

Tachykardie, paroxysmale supraventrikuläre und ventrikuläre 1814

Teflonpfannen, Uberhitzung und Gesundheitsschäden 1815

Testosteron, Plasmaandrogene nach oraler Gabe $\mathbf{1 7 7 3}$

Thallium-201, Myokard-Szintigraphie 2373

Theophyllin-Äthylendiamin, orale broncholytische Langzcittherapic, Lungenfunktion 2482

Thorotrast-Schäden 2126

Thromboembolieprophylaxe, postoperative, mit Dihydroergotamin 2065

Thromboseprophylaxe, Calciumheparinat 2014

Thrombozytopathie, ADP-Freisetzungshemmung ("aspirinlike defect ") 1842

Thrombozytopenien, Autoimmun-, Plättchenproduktion und lincale-speicherung 2641

-, Differentialdiagnose $\mathbf{2 4 9 2}$

-, Therapic 2494

Thyreoiditis de Quervain 2377

Thyroxin-Konversion, exzessive periphere, $\mathrm{T}_{3}$-Hyperthyreose 2319

Tiefgefrieren von Lebensmitteln 2511

Tierhaarallergien, Erkrankungen der oberen Luftwege 2557

Tobramycin, Vergleich mit Gentamicin, Sisomicin 2144

Todesursachenstatistik 1516

Tonsillektomie, bei "Fokaltoxikose" 1871

Transfusion, Bereitstellung von Blut vor Operationen 1997

-, Verweigerung aus religiösen Gründen 2179, 2622

Treitzsche Hernie, angiographische Diagnostik 1902

Trijodthyronin-Hyperthyreose, periphere ThyroxinKonversion 2319

Tropenmedizin, Bedeutung Robert Kochs 1933

Tubenkarzinom, Frïherkennung durch Exfoliativ-Zytologie 1476

Tuberkelbazillenfärbung, 
Tumoren, solide, Chemotherapie, UV-Strahlung, Mutationsgift adjuvante bei -2342

-, urologische maligne, Vorsorgeuntersuchung $\mathbf{2 4 5 2}$

Typhusdauerausscheider, Cholelithiasis 1714

Typhusinfektionen, Verlauf im Raum Heidelberg 2209

\section{$\mathrm{U}$}

Ultraschall, Einfuihrung in die Medirin 2549

Ultraschall-Druckmessung bei arteriellen Rekonstruktionen 1473

Unterkühlung 2132

Ureterocele, kalkdichtes Konkrement 1450

Urinzytologie bei Blasenkarzinom 2340

Urobilinogenprobe bei l.chererkrankungen 1997

Urticaria pigmentosa 1551, 2239

UV-Licht, Einführung in die Medizin 2549

\section{3}

\section{V}

Vacciniaviren, Qualitätsunterschicde, neurale Komplikationen 1457

Vagotomie, Nomenklatur der "dritten" - 2353

Vasodilatatoren bei Gangrän 2658

Vektorkardiographic 2234

Verhaltenshiologic, Beitrag an der psychiatrischen forschung 2108

Vertebralisangiographic, korrikale Blindheir nach 2621

veraugszcit wissenschaftlicher Arbeiten 1716

Vincristin, Sunchronisationstherapic bei malignen Lymphomen 1719

V'irushepatitis A s. Hepatitis A

Vitiligo, nach paratertiärem Butylphenol 2216
Vorhofflattern, atrioventrikuläre Blockicrung 1758

Vorhofflimmern, paroxismales tachvkardes 2389

Vorhoftachykirdie, paroxysmale, A V-Block II. Cirades 1682

Vorsorgeuntersuchung, maligne urologische Tumoren 2452

\section{W}

Warthin-Tumor 1681

Wassermamn-Reaktion, Fintwicklung der 2506

Widal-Reaktion, Vergleich mit indirekter Immunfluoreszen\%, chronische Pyelonephritis 2086

Wolff-Parkinson-White-Syndrom 2536, 2553, 2626)

X

Xeroderma pigmentosum 1821

\section{Z}

Zellvermehrung, Steuerung und Rolle der Zellmembran 2185

Zentralnervensystem, Radio-

Chemotherapie des -, maligne Neoplasien im Kindesalter 1651

Zerebrovaskuläre Insuffizienz, chirurgische Behandlung, extrakranielle Strombahnhindernisse der Arteria cirotis 1823

Zink, Therapie der Acrodermatitis enteropathica 1549

Zuwanderungsbeschränkung für Arzte, Kamada 1583

Zwerchtellparese, genuine 2465

Zystadenolymphom, papilläres 1681

Zytologische Untersuchungen hei Metastasenleber $\mathbf{2 6 0 2}$

Zytostatika, Sensibilitätstestung in vitro bei Mammakarzinom 2035

Zytostatika-Therapie, akute beidseitige Lungeninfiltration als Komplikation 2098

\section{Buchbesprechungen}

\section{Alstrup, P.: Investigation into} Respiratory and Haemodynamic Changes following Pulmonary Surgery with special reference to the aetiology of postoperative atrial fibrillation 2185

Amirow, R. S.: Flektrokardiotopographie 1817

Anacker, H.: Efficiency and Limits of Radiologic Examination of the Pancreas 2412

Battegay, R., U. Rauchfleisch: Medizinische Psychologie 2061

Baumgartl, F., K. Kremer, H. W. Schreiber (Hrsg.): Spezielle Chirurgie in der Praxis 1920

Beck, F., J. B. Lloyd (Editors): The Cell in Medical Science 1817

Bloomfield, D. A.: Dye Curves 2413

Bohlig, H.: Lunge und Pleura 2368

Boschann, H. W.: Ginäkelogische Zytodiagnostik für Klinik und Praxis 2237

Breit, A. (Hrsg.): Wertigkeit radiologischer Methoden. Niere-Leber-Pankreas 2301

Breuer, H., D. Hamel, H.-L. Krüskemper: Methoden der Hormonbestimmung 1999
Burton, A. C.. Phrsiologic et Biophysique de la Circulation 2128

(.hapchal, G.: The Arthrodesis in the Restoration of W'orking Ability 2236

(:ibal Foundation Symposium: The Poisoned Patient: the role of the laboratory 2061

Cooper, P.: Poisoning by Drugs and Chemicals, Plants and Animals 1818

Cou'an/Stecl: Manual for the Identification of Medical Bacteria 2413

Cramer, H., G. Obly: Die Kolposkopie in der Praxis 2184

Crowley, E. V.: An Introduction to Clinical Embryology 2128

Dabm, K., M. Rehner (Hrsg.): Das Karzinom im operierten Magen 2300

Daniels, J., W. Hagen, H. I.ehmkubl, F. Pürckbauer, E. Schröder, J. Stralau, C. L. P. Trüb (Hrsg.): Das öffentliche (iesundheitswesen 2127

Dietel, K.: Die Haut und ihre Frkrankungen im Kindesalter 1873

Dorndorf, $W$.: Schlaganfälle 2235

Drews, G.: Mikrobiologisches Praktikum 2060
Dubots, E. I... Lupus Frvthomatosus 2301

Fpstein, B. S.: The Vertehral Column 2185

Grundmann, F., H. Grunze', S. W'itte: Early Ciastric Cancer 1754

Hafner, F., H. Ch. Meuli: Röntgenuntersuchungen in der Orthopädie 1817

Hager, W., A. Seling: Praxis der Schrittmachertherapie 1754

Hamm, J. (Hrsg.): Interstiticlle Lungenerkrankungen Lungenfibrosen 2184

Handbuch der Inneren Medizin, Bd. 2 Teil 5 1921, Bd. 2/Teil 4 1999

Handbuch der allgemeinen Parhologie, Bd. 92236

Hegglin, R.: Differentialdiagnose innerer Krankheiten 1815

Heisig, N.: Diabetes und Schwangerschaft 1754

Helmclen, H., H. Hippius (Hrsg.): Entwicklungstendenzen biologischer Psychiatrie 2512

Hevden, S.: Gesunde Kost Gesundes Herz 1816

Holluich, F., B. Verbeck: Augen. heilkunde für das Krankenpflegepersonal 2368
Holtmeier, H.- J.: Diät bei Öhergewicht und gesunde Frnährung 1960

Holtmeier, H.-J., L. Weisbecker (llisg.): (hemotherapie der Problemkeime 1816

Hom, H., M. Prib'ora, W. W'euffen: Handbuch der Desinfektion und Sterilisation 2185

Irnich, W.: Finführung in die Bioelektronik 2412

lu'emark, B.: Kinderpathologie 2513

Johnson, F. N.: Lithium Research and Therapy 2237

Kaiser, R.: Hormonale Behandlung von Zyklusstörungen 2301

Keidel, W. (Hrsg.): Kurzgefaßtes Lchrbuch der Physiologie 2512

Krauth, J.: Grundlagen der Marhematischen Statistik für Bio-Wissenschaftler 2061

Kuschinsky, G., R. Lindmur, U. Wollert: Kurs der Allgemeinen Pharmakologie und Toxikologie 1755

Lancin, P. (Hrsg.): Praxis der Intensivbehandlung 2466

I.indenschmidt, Th.-O. (Hrsg.): Pathophysiologische Grundlagen der Chirurgie 2368 
Losse, H., U. Gerlach, F. Wetzels (Hrsg., : Rationelle Therapie in der :nneren Medizin 1754

Mathe, (.. R. Weiner: Investigation and Stimulation of Immurite in (ancer Patients 2467

Mathe, (i., Y. Konis: La (himiotherapse des Cancers 2060

Mlatthes, A.: Epilepsic 2235

()ttenj.mn, R.: (optimierte rationclle Diagnostik in der (iastruenterologic 1817

Perlemuter, l., P. Obraskil, I. () ucrantlliers: Dictionnaire pratique de therapeutique medicale 1816

Pleifier, (.. I., G. Rouden, 1. IX'cibel: Castrointestinal Ultrastructure 2127

Picird, J.-D.: Le Sein The Breast 2513
Raffensperger, J. G., R. A. Seeler, R. Moncada: Das akute Abdomen im Neugeborenen- und Kindesalter 1816

Rajka, G.: Atopic Dermatitis 2467

Ross, E. J.: Aldosterone and Aldosteronism 1755

Silpepank, H.: Erb- und Umweltfaktoren bei Neurosen 1818

Schöll, A.: Therapicergebnisse hei Mamma-Karzinom nach Androgenbehandlung 1921

Sibölmerich, P., H.-P. Sibuster. H. Schönborn, P. P. Banm (Hreg.): Interne Intensivmedizin 1998

Schormiller. J.: Lehrbuch der Lehensmittelchemie 2184

Silraml, W. J., U. B.nmmann (Hrsg.): Klinische P'sychologie 2368
Schuchurdt, K. (Hrsg.): Fortschritte der Kiefer- und Gesichts-Chirurgic 2184

Siggalurd-Anderse'n, ().: The Acid-Base Status of the Blood 2060

Squite, I. F., IW. M. Colctiace, N. Strutynsky': Ubungen in radiologischer Diagnostik 1872

Starck, D.: Embryologic 2059

Steiniger, U., H. Theile (Hrsg.): Funktionsdiagnostik im Kindesilter 2127

Stöfen, I).: Blei als Umweltgift 2237

Teschendorf, $\mathbb{W}^{\prime}$. H. Anacker. P. Thurn: Röntgenologische Differentialdiagnostik 1920

Thomas, (., T. Windt, E. Grom: Hämatologische und endokrine Formen des paraneoplastischen Syndroms 2412
Thome, R., G. Wagner: Dokumentation, Datenverarbeitung und Statistik in der Medizin 1999

Velhagen, K.: Tafeln zur Prüfung des Farbensinnes 1873

Valvassori, G. E., R. A. Buckingham: Tomography and Cross Sections of the Ear 1998

Vorberr, H.: The Breast 1873

IX'ıtzkı, M.: Kurzlehrbuch der Histologie und mikroskopischen Anatomie des Menschen 1873

Wende, S., E. Zieler, N. Nakay'ama: Cerebral Magnification Angiography 2127

Wiendt, H.-J.: Zur Frïhdiagnose des Magenkarzinoms 2236

Zollinger, H. U.: Pathologische Anatomie 2466

\section{Verhandlungsberichte}




\section{Aktuelle Diagnostik}

Redaktion:

Prof. Dr. H. Hornbostel, Hamburg

Prof. Dr. W. Kaufmann, Köln

Prof. Dr. W. Siegenthaler, Zürich
Disch. med. Wschr. 100 (1975), 2223-2225

(C) Georg Thicme Verlag, Stuttgart

\section{Das autonome Adenom der Schilddrüse}

Die früher ausschließlich szintigraphische Diagnostik des autonomen Adenoms ist auch heute noch eine nuklearmedizinische Domäne $(1,9,15,17)$, obwohl die Klärung dieser Fälle durch endokrinologische Methoden vereinfacht und zuverlässiger wurde. Rein klinische Symptome oder histologische Befunde sind nicht pathognomonisch $(14,15)$. Trotz des Altersgipfels nach der vierten Dekade häufen sich die Beobachtungen bei jüngeren Patienten. Eine endokrine Opthalmo- und Dermatopathie (8) schließt ein autonomes Adenom aus. Oligosymptomatische Verlaufsformen, insbesondere mit Herzrhythmusstörungen bei älteren Patienten, sind nicht spezifisch für das hyperthyreote autonome Adenom. Viele autonome Adenome werden erstmals auf Grund sogenannter warmer oder heißer Knoten im ${ }^{99 \mathrm{~m}}$ Technetium-Pertechnetat(oder ${ }^{131}$ Jod-)Szintigramm bei einer Basisuntersuchung (9) wegen Schilddrüsenvergrößerung oder klinisch fraglicher Schilddrüsenfunktionsstörung entdeckt.

Definition: Ein autonomes Adenom ist ein meist knotig tastbares Areal in der Schilddrüse, das unabhängig von der Stimulation durch das thyreotrope Hypophysenvorderlappenhormon (TSH) Schilddrüsenhormone bildet und freisetzt. Solange die Schilddrüsenhormonsekretion aus dem autonomen Adenom eine kritische Grenze nicht übersteigt $(13,15)$, bleibt die thyreotrope Stimulation erhalten. Das paranoduläre Gewebe wird im szintigraphischen Bild durch die Radionuklidspeicherung dargestellt, so daß nach dem Szintigramm vom sogenannten kompensierten (heißer Knoten in einer speichernden Schilddrüse) oder vom maskierten (Struma nodosa ohne deutlich erkennbaren heißen Knoten) autonomen Adenom gesprochen wird. Bei erhöhter Schilddrüsenhormonsekretion aus dem autonomen Adenom wird über den Rückkopplungsmechanismus die endogene TSH-Sekretion dagegen supprimiert, so daß das szintigraphische Bild eines sogenannten dekompensierten autonomen Adenoms durch Ruhigstellung des paranodulären Schilddrüsengewebes entsteht.

Die szintigraphische Einteilung erfolgt nach folgenden Kriterien: Bei einem Impulsratenverhältnis von $100 \%$ im heißen Knoten zu über $20 \%$ im paranodulären Gewebe sprechen wir von einem kompensierten autonomen Adenom. Nimmt das paranoduläre Gewebe weniger als $10 \%$ der Aktivität des heißen Knotens auf, so liegt ein dekompensiertes autonomes Adenom vor. Zwischen $10 \%$ und $20 \%$ Speicherung im paranodulären Gewebe

\section{H. G. Heinze, C. R. Pickardt und P. C. Scriba}

Klinik und Poliklinik für Radiologic (Dircktor: Prof. Dr. J. Lissner) und II. Medizinische Klinik (Direktor: Prof. Dr. E. Buchborn)

der Universität München

sprechen wir von Ưbergangsformen (fast dekompensiertes autonomes Adenom).

Es sind also unterschiedliche Funktionszustände des autonomen Adenoms zu berücksichtigen, die ebenfalls grundsätzlich verschiedene diagnostische Wege erfordern.

\section{Kompensiertes autonomes Adenom}

Dem szintigraphischen Bild entsprechend ist die endogene TSH-Sekretion dieser Patienten normal $(13,16)$. Der Stimulationstest mit $200 \mu \mathrm{g}$ TRH (»thyrotropin releasing hormone «) intravenös führt bei diesen Patienten zu einem normalen TSH-Anstieg (Normalbereich des Anstiegs $30 \mathrm{~min}$ nach Injektion: 2,7 bis $23,6 \mu \mathrm{E} / \mathrm{ml}$ [13]). Die peripheren Schilddrüsenhormonspiegel sind im allgemeinen normal. Diese diagnostischen Verfahren sind also zum Nachweis eines kompensierten autonomen Adenoms ungeeignet.

Der Nachweis der Autonomie des heißen Knotens im speichernden Schilddrüsengewebe erfolgt mit dem Suppressionstest, der zur Unterdrückung des paranodulären Gewebes, nicht aber des Adenoms führt.

Durchfübrung des Suppressionstests: Zur Basisdiagnostik ist jetzt ein Radiojod-Zweiphasentest mit Szintigramm und Impulsratenmessung über dem warmen oder dem heißen Bezirk sowie über dem paranodulären Schilddrüsengewebe zu fordern $(1,9)$.

Erst wenn die Testaktivität aus der Schilddrüse verschwunden ist (nach etwa drei bis vier Wochen, entsprechend vier effektiven Halbwertzeiten), sollte der Suppressionstest begonnen werden, da anderenfalls eine fehlende Suppression im paranodulären Gewebe vorgetäuscht werden kann. Zur Suppression verabreicht man entweder $60 \mu \mathrm{g}$ Trijodthyronin (7 Tage vor und an den 3 Tagen während des zweiten Radiojodtests [4]) oder $3 \mathrm{mg}$ Thyroxin als einmalige Dosis 7 Tage (12) vor dem zweiten Radiojodtest. Auch multiple autonome Adenome kommen, wenn auch selten, auf diese Weise zur Darstellung.

Diese aufwendige Diagnostik ist trotz aller Fortschritte auch heute noch bei Verdacht auf ein kompensiertes autonomes Adenom erforderlich, da die Erfahrung gelehrt hat, daß ein einfaches Suppressionsszintigramm, zum Beispiel mit ${ }^{99 \mathrm{~m}}$ Technetium-Pertechnetat, auf Grund der willkürlichen Einstellung und der subjektiven Beurteilung der Szintigramme häufig zu Fehldiagnosen führt. Besonders Börner und Mitarbeiter (1) haben auf die Schwierigkeit der Diagnostik und die Möglichkeiten zur Fehldiagnose aufmerksam gemacht und auf die Bedeutung von Kontrollszintigrammen nach behandlungs- 
freiem Intervall hingewiesen. Wir halten daher den Vergleich der Änderungen der Radiojodspeicherungskurve und des Impulsraten-Verhältnisses zwischen Adenom und paranodulärem Gewebe nach Suppression für die zuverlässigsten diagnostischen Kriterien des kompensierten autonomen Adenoms.

\section{Dekompensiertes autonomes Adenom}

Stellt sich im normalen Schilddrüsenszintigramm ein isoliert speichernder Knoten dar, ohne daß das paranoduläre Gewebe abgebildet wird, so besteht der Verdacht auf ein dekompensiertes autonomes Adenom, dessen Schilddrüsenhormonproduktion zu einer Suppression der endogenen TSH-Spiegel geführt hat. Dieser Verdacht wird heute bestätigt durch

1. den szintigraphischen Nachweis paranodulären Schilddrüsengewebes und

2. den Nachweis der Suppression der endogenen TSHSpiegel.

3. Durch Bestimmung der Schilddrüsenhormonspiegel im Serum ist die Schilddrüsenfunktion zu klären.

Ad 1. Im Gegensatz zum kompensierten autonomen Adenom verwendet man zum szintigraphischen Nachweis des dekompensierten autonomen Adenoms ${ }^{99 \mathrm{~m}} \mathrm{Tech}$ netium-Pertechnetat. Es bietet den Vorteil der schnelleren Durchführbarkeit, der besseren Zählstatistik auf Grund der höheren verabreichten Aktivität bei gleichzeitig geringerer Strahlenbelastung der Schilddrüse. Zum Nachweis des paranodulären Schilddrüsengewebes empfiehlt sich heute die empfindlichkeitsmodulierte Schilddrüsenszintigraphie (17): Auf Grund einer geringen Restfunktion der nicht autonomen und daher "supprimierten " Schilddrüsenanteile gelingt es, auch diese Anteile szintigraphisch sichtbar zu machen, wenn das Impulsmaximum des Scanners um 50 bis $100 \%$ über der Impulsrate des Untergrundes (also einer Region außerhalb des Schilddrüsenlagers) eingestellt wird. Dabei wird die Abtastgeschwindigkeit verringert und zur Schonung des Schreibwerkes darauf verzichtet, den schon aus der Erstuntersuchung bekannten heißen Knoten erneut mit darzustellen. Zur Vermeidung von Uberstrahlungen in der Randregion des heißen Knotens wird bei großer Impulsratendifferenz zwischen dem heißen Knoten und den paranodulären Gewebeanteilen vorteilhafterweise ein hochenergetischer, hochauflösender Kollimator auch bei ${ }^{99 \mathrm{~m}}$ Technetium-Pertechnetat verwendet.

Bei Anwendung der empfindlichkeitsmodulierten Szintigraphie kann eine kugelige Schilddrüse oder eine einseitige Schilddrüsenaplasie ausgeschlossen werden. Damit kann auf die aufwendige und nicht gefahrlose Stimulation der Schilddrüse durch exogenes TSH (TSHStimulationstest, Stimulations-Szintigramm [3]) verzichtet werden, zumal die TSH-Injektion zu einer eventuell kritischen Erhöhung der Schilddrüsenhormonspiegel führen kann, da auch das autonome Adenom mit einer Schilddrüsenhormonfreisetzung auf TSH reagiert $(3,7)$.

Der Nachweis fehlender Supprimierbarkeit des heißen Knotens durch einen Suppressionstest ist ebenfalls nicht ungefährlich, da zu der oft bestehenden Erhöhung der Schilddrüsenhormonspiegel eine zusätzliche exogene Schilddrüsenhormonbelastung hinzukommt. Dies ist auch nicht indiziert, da die endogene TSH-Sekretion definitionsgemäß bereits supprimiert ist.

Ad 2. Zum Nachweis der Suppression der TSH-Sekretion dient heute der TRH-Stimulationstest: Steigen die niedrigen basalen TSH-Spiegel nicht an, so ist die Suppression der TSH-Sekretion nachgewiesen (13).

Ad 3. Diese Suppression der TSH-Spiegel bedeutet jedoch noch nicht, daß alle Patienten zum Zeitpunkt der Untersuchung eine Hyperthyreose haben $(2,5,6,13)$. Funktionell zeigen sich in der Gruppe der Patienten mit einem dekompensierten autonomen Adenom drei verschiedene Zustandsbilder, nämlich zunächst die Patienten mit einer klassischen Hyperthyreose mit erhöhten Thyroxin- und erhöhten Trijodthyronin-Spiegeln. Die zweite Gruppe umfaßt Patienten, die bei normalen oder erniedrigten Thyroxinspiegeln (7) und normalem thyroxinbindendem Globulin im Serum erhöhte Trijodthyroninspiegel haben, also eine sogenannte TrijodthyroninHyperthyreose. Die dritte Gruppe umfaßt Patienten mit dekompensierten autonomen Adenomen, die zum Zeitpunkt der Untersuchung normale Thyroxin- und Trijodthyronin-Spiegel haben (persistierende Suppression oder phasenhafter Verlauf [13]). Da diese drei Zustandsbilder klinisch mit Ausnahme der klassischen Hyperthyreose nur bei gleichzeitiger Bestimmung der Trijodthyroninspiegel $(5,6)$ voneinander $\mathrm{zu}$ unterscheiden sind, sollte die Diagnose "toxisches Adenom ", anhand des szintigraphischen Bildes gestellt, nicht mehr verwendet werden $(13,16)$.

Tabelle 1 gibt eine Ưbersicht über den Untersuchungsablauf.

Tab. 1. Untersuchungsablauf

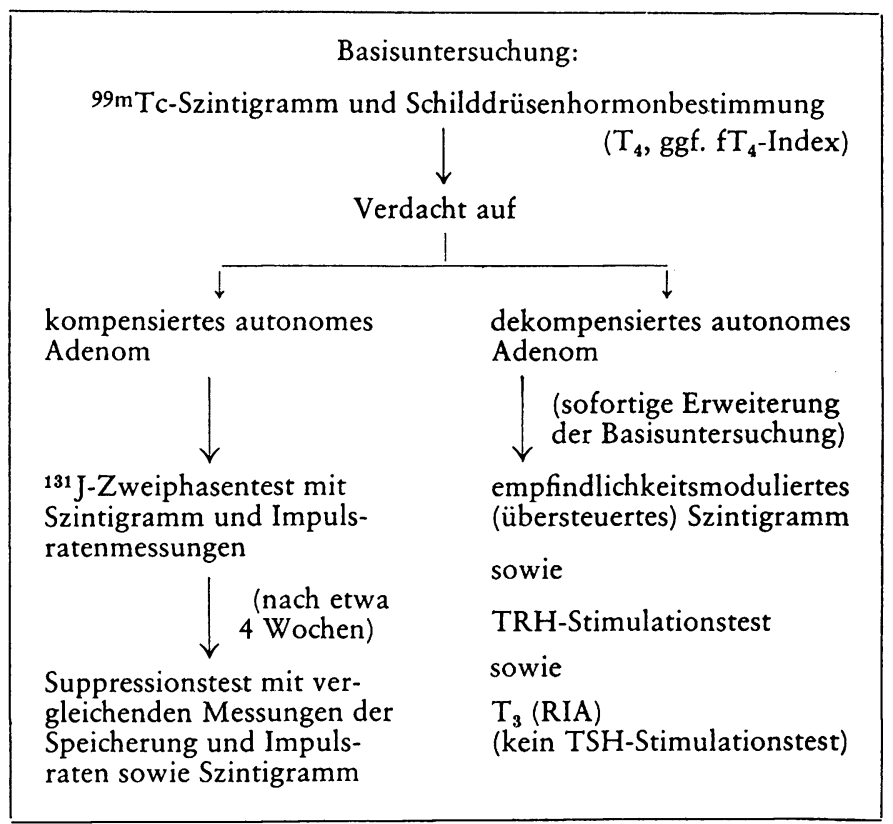

Mit der zu erwartenden Verbreitung von Szintillationskameras mit Auswertesystemen wird der zuverlässige Nachweis kompensierter autonomer Adenome auch mit der quantitativen Szintigraphie sowie kurzlebigen Isotopen des Jods bzw. mit $99 \mathrm{mTc}$ möglich. 


\section{Differentialdiagnostische Probleme}

Auch bei Vergleich der Impulsraten über dem heißen Areal und dem paranodulären Schilddrüsengewebe kann die Abgrenzung einer hyperthyreoten Knotenstruma ohne Ophthalmopathie von einem in Dekompensation begriffenen autonomen Adenom problematisch sein, nämlich dann, wenn das paranoduläre Gewebe zwischen 10 und $20 \%$ der Aktivität über dem heißen Knoten aufnimmt. Dieser Übergang kann mit normalen, aber auch mit erhöhten Schilddrüsenhormonspiegeln und schon supprimierter TSH-Sekretion einhergehen (13). Ein TSH-Stimulationstest bringt in diesen Fällen das bereits szintigraphisch nachweisbare paranoduläre Gewebe lediglich deutlicher zur Abbildung. Ein $\mathrm{T}_{3}$-Suppressionstest ist aus klinischen Gründen in der Regel zu risikoreich und bei bereits supprimierter TSH-Sekretion auch nicht indiziert.

Ferner fällt nach Jodexposition (Kontrastmittel) oft die differentialdiagnostische Entscheidung schwer, ob primär eine blande Struma nodosa mit sekundärem, eventuell transitorischem "Jod-Basedow « oder ob primär ein autonomes Adenom vorgelegen hat, da nach Jodapplikation einerseits das szintigraphische Bild qualitativ schlecht und schwer deutbar und andererseits die TSH-Sekretion supprimiert sein kann.

Fehldiagnosen können bei sogenannten maskierten autonomen Adenomen unterlaufen, bei denen das autonome Adenom in der Struma nicht als heißer Knoten in Erscheinung tritt. In diesen Fällen kann ein Suppressionstest die Diagnose klären, während die TSH-Sekretion nach unseren Ergebnissen (13) im Gegensatz zu anderen Untersuchern (5) sowohl bei blanden Knotenstrumen als auch bei kompensierten (maskierten) autonomen Adenomen stimulierbar ist. Häufig genug wird jedoch das autonome Adenom erst im Verlauf einer Schilddrüsenhormonbehandlung der vermeintlich blanden Struma nodosa oder nach Jodexposition erkannt. In Zweifelsfällen empfiehlt es sich, bei diesen Patienten eine engmaschige Kontrolle anzuschließen (1), insbesondere in Anbetracht der phasenhaften Funktionsänderungen bei autonomen Adenomen.

Jodexpositionen von Patienten mit autonomen Adenomen führen zum Anstieg der Schilddrüsenhormonspiegel und zur Dekompensation (11). Es wäre daher naheliegend, einen Jodbelastungstest anzuwenden (10),

wenn dieses Verfahren wegen des Hyperthyreoserisikos vertretbar wäre.

Ist die Diagnose eines kompensierten oder dekompensierten autonomen Adenoms gestellt, so ist die konsequente Ausschaltung dieses autonomen Areals operativ oder durch Radiojodbehandlung indiziert, da eine spontane Rückbildung $(13,16)$ nur in sehr seltenen Fällen zu erwarten ist.

\section{Literatur}

(1) Börner, W., E. Moll, E. Rauh, A. Pohner, S. Grehn, G. Ruppert: Diagnostik des autonomen Adenoms de Schilddrüse. Drsch. med. Wschr. 96 (1971), 1707.

(2) Botsch, H., B. Schreiber, K. Oeff: Serumkonzentration des Trijodthyronins beim autonomen Adenom der Schilddrüse. Münch. med. Wschr. 114 (1972), 2181.

(3) Heinze, H. G., J. Wöhler,

H. Ingrisch, K. J. Pfeifer, I.

Souvatzoglou, K. Horn, P. C. Scriba: TSH-Stimulation mit niedrigen Dosen zur Diagnostik des autonomen Adenoms - Treffsicherheit und Risiko des TSH-Tests. Disch, med. W'schr. $99(1974), 1236$

(4) Heinze, H. G., H. W. Pabst, J. Klemm, G. Hör: L-TrijodthyroninSuppressionstest der Schilddrüsc. Therap. Umsch:au 24 (1967), 397. (5) Hesch, R.-D., D. Emrich, A. v. zur Mühlen, H.-P. Breuel: Der Aussagewer der radioimmunchemischen Bestim mung von Trijodthyronin und thyreotropem Hormon für die Schilddrüsendiagnostik in der Praxis. Disch. med. Wschr. 100 (1975), 805

(6) Horn, K.: Trijodthyronin. Probleme der Bestimmung, pathophysiologische und diagnostische Bedeurung. Habilitationsschrift, München 1975. (7) Ingrisch, H. H. G. Heinzc, J. Wöhler, K. Horn, K. J. Pfeifer, P. C. Scriba: Absolute Jodaufnalsme auto nomer Adenome der Schilddrüse vor und nach exogener TSH-Stimulation. Disch. med. Wschr. 99 (1974), 1677.

(8) Klcin, E., J. Kracht, H. L. Krïskemper, D. Reinwein, P. C. Scriba Klassifikation der Schilddrüsenkrankheiten. Dtsch. med. Wschr. 98 (1973). 2249.

(9) Klein, E., J. Kracht, H.-L. Krïs

\section{Privatdozent Dr. H. G. Heinze}

Klinik und Poliklinik für Radiologie der Universität 8 München 2, Ziemssenstr. 1

Privatdozent Dr. C. Renate Pickardt, Prof. Dr. P. C. Scriba II. Medizinische Klinık der Universität 8 München 2, Ziemssenstr. 1 kemper, D. Reinwein, P. C. Scriba: Praxis der Schilddrüsendiagnostik. Disch. med. Wschr. 98 (1973), 2362 (10) Kutzim, H., A. Magundar U. Wellner: Provokationstest zur weiteren Differenzicrung der Struma. In: Victen, H. (Hrsg.): Deutscher Röntgenkongreß 1971 (Thiemc: Stuttgart 1972) 159

(11) Mahlstedt, J., K. Joseph: Dekompensation autonomer Adenome der Schilddriise nach prolongierter Jodzufuhr. Disch. med. Wschr. 98 (1973), 1748

(12) Mahlstedt, J., K. Joscph, F. H. Graul: Suppressionstest der Schilddrüse nach einmaliger Gabe von $3 \mathrm{mg}$ L-Thyroxin. Nuc-Compact (1972), 4. (1.3) Pickardt, C. R., F. Erhardt, J. Grïner, H. G. Heinze, K. Horn, P. C. Scriba: Stimulierbarkeit der TSH-Sekretion durch TRH bci autonomen Adenomen der Schilddrüse. Disch. med. Wschr. 98 (1973), 152. (14) Pohl, G., G. Galvan, H. Stciner, R. Salis-Samaden: Das autonome Adenom der Schilddriise im Struma Endemicachict. Disch med W/schr. $98(1973), 189$.

(15) Schncider, C., Kl. J. Thiemann, V. Bay: Dic Symptomatik des toxischen Adenoms der Schilddrüse in verschicdenen Lebensaltern. Disch. med. Wschr. 9.5 (1970), 387

(16) Wenzel, K. W., H. Meinhold,

H. Schleusener, H. Botsch: Verbesserte Beurteilungskriterien des autonomen Adenoms der Schilddrïse: Trijodthyro nin-Konzentration im Serum, funktionelle Definition durch den TRH-Test. Dtsch. med. Wschr. 99 (1974), 1465. (17) Wöhler, J., H. G. Heinze, C. R. Pickardt, F. Erhardt, P. C. Scriba: Eine neue, risikolose Methode zur Diagnostik dekompensierter autonomer Adenome der Schilddrïse. Dtsch. med. Wschr. 99 (1974), 1240. 